\title{
SCINTILLATING LENSES
}

\author{
M. A. WALKER
}

Research Centre for Theoretical Astrophysics, A28

School of Physics, University of Sydney, NSW 2006, Australia

\section{Introduction}

There are now several examples of distant radio sources being gravitationally lensed by foreground galaxies (e.g. PKS1830-211, Jauncey et. al. 1991). In such cases we know that the line of sight must be passing through a substantial amount of ionized material, so it is pertinent to ask whether these free electrons influence the observed properties of the source in any significant way.

\section{Smooth Lenses}

Firstly the ionized gas in the lensing galaxy/cluster behaves on average as a smooth lens which alters the appearance of any background radio source. Hence one expects that the plasma lens ought to be accounted for when modeling the radio image structure of any gravitationally lensed source. In practice though, such effects are very weak indeed and the smooth plasma lens can be ignored in most circumstances. (Just because the contribution of gravity to the interstellar refractive index is typically much larger than the free-electron contribution, at observable radio wavelengths.)

This seems to be at odds with the well known influence of the ISM on radio wave propagation in our Galaxy contrasted with the apparent lack of any observational consequences of the gravitational field. This situation has arisen because of time, frequency or polarization dependence in the plasma's influence, whereas it is well known that gravitational lensing is achromatic and polarization-independent. However, it is also the case that time dependence of the lensing geometry is offset by the long-range nature of the gravitational field. In contrast, the influence of a plasma is completely localized to those rays which pass through it; changes in the viewing geom- 
etry then lead to the phenomenon of interstellar scintillation as a result of inhomogeneities in the plasma.

\section{Rough Lenses}

Quite generally one can think of the inhomogeneities in the plasma as introducing a distorting, transparent screen - a phase screen - between source and observer. We can immediately identify a number of interesting phenomena arising from the presence of such a phase screen: we expect a complex, evolving pattern of magnifications across the screen; magnifications will be different for small vs. large sources - even if they are coincident - for the same source at different wavelengths, and different again for identical sources in different locations; images will be seen at (wavelength dependent) locations slightly different from those which would be recorded in the absence of the phase screen.

Applying scintillation theory to the case of gravitationally lensed extragalactic sources raises the following points concerning the effect of the ISM in our own Galaxy. Extragalactic sources are too great in angular size to exhibit diffractive scintillation, but often show slow refractive scintillations; even then, only the cores of these sources are sufficiently small to show scintillations - jets and lobes should have no significant magnification introduced by the ISM. For multiple images separated by $\sim$ arcseconds, the scintillations should be essentially independent of each other (as this is much larger than the angular scale of the ISM irregularities which are causing the brightness variations). This is a source of noise in establishing the relative time delay between images. If refractive scintillations are significant then there should also be an associated image (scatter) broadening; temporal smearing associated with the image broadening is negligible.

We can extrapolate this theory into the extragalactic realm if we simply assume that the ISM in the lensing galaxy is similar to that in our own Galaxy. (This is probably not realistic as many lenses seem to be elliptical galaxies, whose ISM is much more homogeneous than spirals.) Using this assumption one finds that the refractive scintillations introduced by a lensing galaxy are typically expected to be small (less than $10 \%$ for frequencies above $1.4 \mathrm{GHz}$ ). Further, the scintillation time-scale is very long - in excess of a thousand years - so the plasma lens can be regarded as static. Angular broadening of compact sources might, however, be measurable.

\section{References}

Jauncey D. L. et al. 1991, Nature, 352, 132 